\title{
A review of the clinical utility of duloxetine in the treatment of diabetic peripheral neuropathic pain
}

\author{
This article was published in the following Dove Press journal: \\ Therapeutics and Clinical Risk Management \\ 10 August 2015 \\ Number of times this article has been viewed
}

\section{Jordan B King \\ Marisa B Schauerhamer \\ Brandon K Bellows}

Pharmacotherapy Outcomes Research Center, University of Utah College of Pharmacy, Salt Lake City, UT, USA
Correspondence: Brandon K Bellows University of Utah College of Pharmacy, 30 South 2000 East, Room 4963, Salt Lake City, UT 84II2, USA

$\mathrm{Tel}+\mathrm{I} 80 \mathrm{I} 58780$ I I

Fax +I 80I 5877923

Email brandon.bellows@pharm.utah.edu
Abstract: Diabetes mellitus is a world-wide epidemic with many long-term complications, with neuropathy being the most common. In particular, diabetic peripheral neuropathic pain (DPNP), can be one of the most distressing complications associated with diabetes, leading to decreases in physical and mental quality of life. Despite the availability of many efficient medications, DPNP remains a challenge to treat, and the optimal sequencing of pharmacotherapy remains unknown. Currently, there are only three medications approved by the US Food and Drug Administration specifically for the management of DPNP. Duloxetine (DUL), a selective serotonin-norepinephrine reuptake inhibitor, is one of these. With the goal of optimizing pharmacotherapy use in DPNP population, a review of current literature was conducted, and the clinical utility of DUL described. Along with early clinical trials, recently published observational studies and pharmacoeconomic models may be useful in guiding decision making by clinicians and managed care organizations. In real-world practice settings, DUL is associated with decreased or similar opioid utilization, increased medication adherence, and similar health care costs compared with current standard of care. DUL has consistently been found to be a cost-effective option over short time-horizons. Currently, the long-term cost-effectiveness of DUL is unknown. Evidence derived from randomized clinical trials, real-world observations, and economic models support the use of DUL as a first-line treatment option from the perspective of the patient, clinician, and managed care payer.

Keywords: clinical trials, pharmacoeconomic studies, opioid-utilization, health care utilization, pregabalin, tricyclic antidepressants, gabapentin

\section{Introduction}

Twenty-one million persons in the United States are currently diagnosed with diabetes mellitus and an additional 8.1 million persons are suspected to be undiagnosed. ${ }^{1}$ In 2012, there were 1.7 million new cases of diabetes diagnosed in United States adults. ${ }^{1}$ Patients with diabetes are at risk for macrovascular complications such as myocardial infarction and stroke, and microvascular complications such as nephropathy, retinopathy, and neuropathy. It has been estimated that $26 \%-47 \%$ of patients with diabetes have diabetic peripheral neuropathy, which can result in a loss of sensation or pain. ${ }^{2,3}$ Diabetic peripheral neuropathy tends to occur in an ascending fashion, with the nerves in the feet most often affected first. ${ }^{4}$ Loss of sensation may lead to sores or infection in the feet, which, if left undetected by patients, may lead to lower limb amputation. In contrast to loss of sensation, diabetic peripheral neuropathic pain (DPNP) is associated with burning, tingling, shock-like, or shooting pain in the extremities. ${ }^{5,6}$ While it is unknown how many patients suffer from DPNP, it has been reported that $26.8 \%$ of patients with diabetes experience pain or tingling. ${ }^{2}$

According to the American Diabetes Association guidelines, the best way to prevent or slow the progression of neuropathy is to maintain proper glucose control. ${ }^{7}$ 
Prevention and slowing the progression of DPNP via glucose control has been demonstrated to be effective in patients with type 1 diabetes, but may not be as effective in patients with type 2 diabetes. ${ }^{8}$ When adequate glucose control cannot be reached or does not alleviate DPNP symptoms, treatment with pharmacologic agents is warranted. ${ }^{9}$ While only three drugs, pregabalin (PRE), tapentadol, and duloxetine (DUL), have been approved by the US Food and Drug Administration (FDA) specifically for use in management of DPNP, many other drugs have shown to be efficacious for relieving DPNP. ${ }^{10}$ Generally, guidelines recommend using DUL, gabapentin (GABA), PRE, and tricyclic antidepressants (TCAs) as first-line pharmacologic agents for alleviating DPNP.9,11,12 Due to many available options for managing DPNP, it is helpful to compare the benefits of each therapy to determine which may be the best for patients. Clinical utility is one measurement that can be used to compare DPNP agents.

Clinical utility can be broadly defined as the relevance and usefulness of an intervention in patient care. ${ }^{13}$ Assessment of the clinical utility of a drug should encompass benefits, risks, and value for stakeholders in order to aid in the decision making process. Clinical trials and observational studies can be a useful means in assessing clinical utility by clinicians and managed care. Decision makers commonly use evidence derived from clinical trials, as they tend to be less vulnerable to threats of internal validity, and allow for a causal relationship of treatment and effect to be inferred from the results. Clinical trials, however, often suffer from limited external validity, as they are designed to demonstrate efficacy, ie, how an intervention works under ideal conditions. The use of observational studies to aid in decision making is not commonly used as they are susceptible to biases that limit the ability to make causal statements from the results. Contrary to the rigorously controlled clinical trial, observation research establishes associations, not causation. However, this serves to examine the effectiveness of a treatment, ie, how an intervention works in real-world settings. In real-world settings, patients may have comorbidities that would exclude them from clinical trials or may not be adherent to treatment. Also, less commonly used by decision makers when assessing clinical utility, are pharmacoeconomic studies, which quantify the costs and benefits of drug therapies to determine value. To better understand the clinical utility of drug therapy, clinical trials, observational studies, and pharmacoeconomic studies need to be considered together to answer the questions can it work, does it work, and is it worth it?
The objective of this paper is to describe the clinical utility of DUL compared to other treatments for DPNP by reviewing the current literature surrounding the clinical efficacy, realworld effectiveness, and cost-effectiveness of DUL in DPNP. This review will also discuss the challenges associated with sources used for obtaining clinical utility information in order to make clinical and managed care decisions.

\section{Literature search and identification}

MEDLINE searches were conducted between December 2014 and April 2015 to identify the current literature regarding the use of DUL for DPNP (Supplementary materials, Table S1). Only studies that were written in English were included in this review. Clinical trials must have included patients with DPNP and a DUL treatment arm in order to be included in this review. Real-world studies were included if they compared the effectiveness or cost of DUL to any other drug treatment for DPNP. Any pharmacoeconomic studies that included evaluation of DUL for the purpose of treating DPNP were also included. Overall, 13 clinical trials, nine real-world studies and five pharmacoeconomic studies were found for inclusion in this review. The authors reviewed these studies and relevant study design, methodology, and outcome information was extracted and synthesized for this paper.

\section{Clinical trials}

All of the clinical trials examined adult patients but differed in minimum duration of DPNP required, as well as the method used to assess DPNP. Comparators to DUL included placebo (PCB), routine care, PRE, GABA, amitriptyline (AMI), and differing doses of DUL. Of the 13 clinical trials, eleven required patients to have DPNP for at least 6 months, and a Michigan Neuropathy Screening Instrument score $\geq 3$ and/or $\geq 4$ on a $0-10$ Likert pain scale. ${ }^{14-24}$ Two trials did not have these same inclusion criteria; one did not specify the duration of DPNP, but used a Leeds Assessment of Neuropathic Symptoms and Signs score $>12,{ }^{25}$ and the other required patients to have DPNP for at least 1 month with a visual analog scale (VAS) pain score $>50 \%{ }^{26}$ The duration of the studies ranged from 4 weeks to 52 weeks. The primary outcomes in eleven of the trials were pain scores, ${ }^{14-16,18-23,25,26}$ while quality of life scores were the primary outcome used by the other two trials. ${ }^{17,24}$ The primary pain outcome was an 11-point Likert scale, used to measure average daily pain, in eight of the studies, ${ }^{14-16,19-23}$ the Brief Pain Inventory (BPI) scale in two studies, ${ }^{18,25}$ and a VAS of 0-100 in the remaining study. ${ }^{26}$ Other outcomes included use of concomitant analgesics, ${ }^{15,16,20,23}$ 
and treatment emergent adverse events. ${ }^{12-16,18-22,24}$ A review of the methods and results of all clinical trials can be found in Table S2.

\section{Pain scores DUL vs PCB}

Pain score reduction of DUL was compared to PCB in five studies, of which four used an 11-point Likert scale $15,16,19,23$ and one used a change in BPI, ${ }^{14}$ to assess efficacy at follow-up. Pain score reductions ranged from 2.4 to 2.9 points with DUL across the four studies using a 24-hour average pain score on an 11-point Likert scale. DUL was found to be significantly better at reducing pain scores than PCB at 8-12 weeks in four of the five studies when DUL was prescribed in doses of at least $60 \mathrm{mg}$ per day. ${ }^{15,16,19,23}$ Goldstein et al found no difference in pain score reduction between DUL $20 \mathrm{mg}$ per day and PCB. ${ }^{15}$ Goldstein et al concluded the difference may be due to a PCB-response that may be prevalent in their patient population due to ethnicity, cultural heritage, comorbidities, possible fluctuation of pain from day to day, and the use of subjective measures. ${ }^{15}$ Gao et al did not find a significant difference between DUL $60 \mathrm{mg}$ or $120 \mathrm{mg}$ and PCB at 12 weeks, but did up to week $4 .^{14}$

Overall, it appears that DUL is favored above PCB in reducing pain in patients with DPNP.

\section{DUL vs PRE}

DUL was compared to PRE in three studies, two of which used an 11-point Likert scale to compare change in pain. ${ }^{21,22,25}$ Pain score reductions with DUL ranged from 2.3 to 2.6 using a Likert scale pain ${ }^{21,22}$ and final BPI severity scores ranged from 2.2 to $2.5 .{ }^{25}$ Tanenberg et al conducted a non-inferiority trial over 12 weeks and found DUL to be non-inferior to PRE with pain score reductions of -2.6 and $-2.1(P=0.08)$, respectively. ${ }^{21}$ Tesfaye et al compared DUL $60 \mathrm{mg}$ to PRE $300 \mathrm{mg}$ over an 8-week period, after which, if a satisfactory reduction in pain was not achieved, patients started on either high-dose monotherapy (DUL $120 \mathrm{mg}$ or PRE $600 \mathrm{mg}$ ) or combination therapy (DUL $60 \mathrm{mg}+$ PRE $300 \mathrm{mg}$ ). ${ }^{22}$ During the initial 8 weeks of treatment, DUL had significantly greater reductions in pain score than PRE (DUL 2.3 vs PRE 1.7, $P<0.001$ ), but no difference was observed between high-dose monotherapy and combination therapy. Boyle et al measured BPI severity after treatment with DUL, PRE, or AMI for 28 days. ${ }^{25}$ Pain scores were reduced with each treatment, but there were no significant differences between comparators. Final BPI severity measurements ranged from 2.2 to 2.5 with DUL $60 \mathrm{mg}$ to $120 \mathrm{mg}$, and 2.3 to 2.4 with PRE $150 \mathrm{mg}$ to $300 \mathrm{mg}$.
Overall, because studies had conflicting results on an increased benefit with DUL when compared to PRE, it cannot be concluded if one drug is superior to the other at reducing pain in DPNP.

\section{DUL vs TCAs}

Two studies were identified that compared DUL to a TCA, both of which used AMI. ${ }^{25,26}$ As previously mentioned in the section DUL vs PRE, Boyle et al measured BPI severity after treatment with DUL, PRE, or AMI for 28 days, and found no significant differences between the drugs. ${ }^{25} \mathrm{BPI}$ severity measurements ranged from 2.2 to 2.5 with DUL $60 \mathrm{mg}$ to DUL $120 \mathrm{mg}$, and 2.6 to 2.7 with AMI $50 \mathrm{mg}$ to $75 \mathrm{mg}$. Kaur et al examined pain reduction on a VAS of $0-100$, which differed from the $0-10$ scale used in the majority of studies in this paper, over 6 weeks in patients in India. ${ }^{26}$ In this study they compared DUL (20 mg to $60 \mathrm{mg}$ per day) to AMI (10 mg to $50 \mathrm{mg}$ per day). They found that both treatments significantly reduced pain at 6 weeks ( $P<0.001$ for both), but there was no difference in the proportion of patients with a VAS reduction of $>50 \%$ (DUL $59 \%$ vs AMI 55\%, $P=$ not significant).

Overall, it appears that DUL and AMI may have similar efficacy, but both of these studies had relatively small sample sizes and used a range of doses, thus, they may have been underpowered to detect differences (Table 1).

\section{DUL dose effects}

Many of the studies reviewed included different doses of DUL. ${ }^{15,16,18,20,22,23}$ In the majority of these, different doses of DUL were compared to PCB, but not to each other. Raskin et al conducted an open-label safety study that also used BPI severity to assess DUL $120 \mathrm{mg}$ per day given as either a single dose or divided into two doses. ${ }^{18}$ They only reported the changes in BPI graphically (estimated to be a reduction of 2.7 for both doses), but they found significant reductions from the baseline score ( $P<0.001$ for both).

Overall, when examining the various doses of DUL used in the studies, doses of $\geq 60 \mathrm{mg}$ per day resulted in significant reductions in pain scores, while doses $<60 \mathrm{mg}$ did not. ${ }^{15,16,18,20,22,23}$

\section{Concomitant analgesic use}

Four studies reported analgesic use by patients in clinical trials. ${ }^{15,16,20,23}$ Raskin et al and Wernicke et al both evaluated the median average daily dose (mg) of acetaminophen used in PCB, DUL $60 \mathrm{mg}$, and DUL $120 \mathrm{mg} .{ }^{16,23}$ Both studies found that only DUL $120 \mathrm{mg}$ was associated with a significant decrease in acetaminophen use compared to PCB; neither 


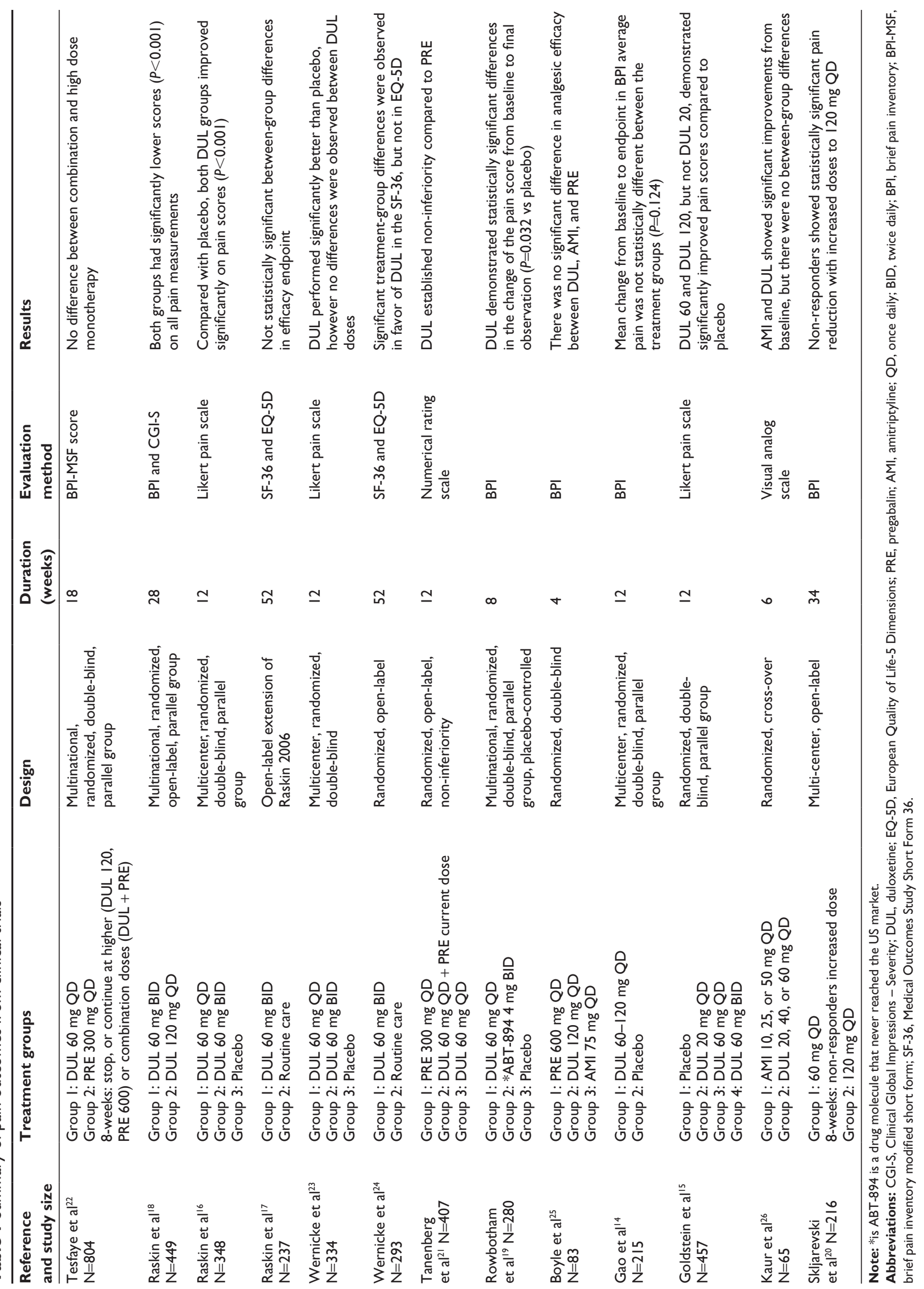


study found a significant decrease in acetaminophen use in patients using DUL $60 \mathrm{mg}$. Goldstein et al did observe a significant reduction in analgesic use with $60 \mathrm{mg}$ of DUL per day, but did not find a significant difference in patients using $20 \mathrm{mg}$ per day. ${ }^{15}$ Skljarevski et al only reported the number of patients using acetaminophen during the study. ${ }^{20}$ Of those using DUL $60 \mathrm{mg}$ or $120 \mathrm{mg}$, eleven (9.6\%) and six $(8.7 \%)$ reported acetaminophen use. ${ }^{20}$

Overall, it appears that use of DUL at $120 \mathrm{mg}$ per day, and possibly $60 \mathrm{mg}$ per day, is associated with reductions in acetaminophen use.

\section{Treatment emergent adverse events}

Treatment emergent adverse events that occurred in 5\% or more of patients in the reviewed studies included: nausea $(2.7 \%-42.6 \%)$, dizziness $(5.5 \%-23 \%)$, somnolence $(1.4 \%$ $26.5 \%)$, headache $(4.1 \%-15.6 \%)$, dry mouth $(5.2 \%-24 \%)$, hyperhidrosis $(1.2 \%-13.9 \%)$, anorexia $(2.6 \%-10.4 \%)$, vomiting $(3.6 \%-16 \%)$, constipation $(8.7 \%-37 \%)$, peripheral edema $(1.4 \%-5 \%)$, fatigue $(5.1 \%-12.5 \%)$, asthenia $(1.2 \%-0.8 \%)$, diarrhea $(3.2 \%-11.4 \%)$, nasopharyngitis $(6.4 \%-7.0 \%)$, and insomnia $(5.3 \%-15 \%)$.

\section{Real-world studies}

Clinical trials are the gold standard for establishing causation between a therapy and an outcome, however, strict inclusion criteria and rigid protocols limit the generalizability of trial results to every day clinical practice. It is increasingly accepted that results drawn from clinical trials are not always useful for decision-making. ${ }^{27}$ Real-world evidence refers to research based on data that is collected in actual practice. This provides a different approach to understanding the benefits and harms of treatment strategies. Unlike clinical trials, real-world patients tend to be more heterogeneous and more closely represent the patients typically seen by physicians in clinic. Hence, the primary strength of real-world evidence is found in the name; real-world studies reflect real-life situations.

A challenge associated with the use of historical, realworld data is a lack of clinical trial efficacy endpoints available within the data. This is particularly problematic in comparative-effectiveness pain research. Clinical trials commonly use pain scales as a measurement of efficacy, however, pain scales are rarely available to the observational researcher in such quantity to make their use feasible as an effectiveness outcome. Instead, surrogate markers of effectiveness that are readily available have been adopted. These surrogates include opioid-utilization, ${ }^{28-30}$ medication adherence as measured by mean medication possession ratio (MPR), ${ }^{31,32}$ health care utilization or costs, ${ }^{28-30,32-36}$ and initiation of additional nonopioid pain related pharmacotherapies. ${ }^{34}$

In total, nine studies evaluating the comparativeeffectiveness of DUL vs other pharmacotherapies in the treatment of DPNP, were identified from the literature search. Studies were published between 2010 and 2013. Eight studies

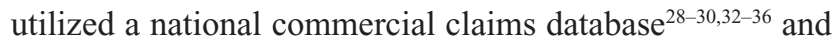
one used the Texas Medicaid database. ${ }^{31}$ All studies required a pre-index and post-index continuous enrollment period and identified patients with medical claims for DPNP (ICD-9-CM $250.6 x, 357.2 x)$. The three most common endpoints were opioid utilization patterns, medication adherence, and changes in health care utilization or costs. A comprehensive review of all real-world studies can be found in Table S3.

\section{Opioid-utilization \\ DUL vs standard of care medications}

DUL was compared to standard of care (SOC) medications (ie, TCAs, venlafaxine, GABA, and PRE), in three studies that had opioid utilization as a primary effectiveness outcome. ${ }^{28-30}$ Chen et al and Wu et al performed very similar historical cohort analyses among patients starting treatment for DPNP with either DUL or SOC in patients not currently receiving opioids. ${ }^{28,29}$ A significantly lower proportion of DUL patients had opioid use than SOC medications after starting DPNP treatment was reported by Chen et al (DUL $52.1 \%$ vs SOC $84.6 \%, P<0.05$ ), and Wu et al (DUL 54.0\% vs SOC $76.7 \%, P<0.05)$. Wu et al also found DUL to be associated with significantly lower adjusted odds of opioid use when compared to SOC (odds ratio 0.38, $P<0.05$ ). ${ }^{28,29}$

In the third study, which was also a historical cohort study, Zhao et al further divided patients based on a MPR above or below $0.8 .^{30}$ This allowed the researchers to determine if adherence, or continuous vs non-continuous medication use, modifies the effect of DUL on opioid utilization in patients currently receiving opioids. There were no significant differences between continuous DUL use and non-continuous DUL, continuous SOC, or non-continuous SOC with regard to number of DPNP-related opioids fills or in cumulative morphine equivalents in the year after starting treatment. It should be noted that the patient population for all three of these studies were drawn from the same database (Medstat Marketscan) during the same index time period (March 2005-December 2005). Additionally, the only meaningful differences between the Chen et al and $\mathrm{Wu}$ et al studies is that the former used propensity score matching, while the latter used multivariable regression. These studies were all funded by Eli Lilly and Company. 
Overall, for patients not currently receiving opioids, starting DUL compared to SOC may lead to fewer patients receiving opioids. However, for patients currently receiving opioids, DUL does not appear to reduce opioid utilization compared to SOC.

\section{DUL vs PRE}

There were two studies that examined opioid utilization for DUL and PRE. ${ }^{34,36}$ Gore et al found no difference in the proportion of patients using opioids prior to and after starting treatment within each group. ${ }^{34}$ Both PRE and DUL had a statistically significant increase in the number of any opioid prescriptions dispensed after starting treatment compared to before (PRE 7.0 vs 7.3, $P=0.01$; DUL 8.5 vs 9.0, $P<0.001)$.

Margolis et al compared utilization of opioid and nonopioid analgesics between DUL and PRE in a historical cohort analysis. ${ }^{36}$ Patients newly initiated on either DUL or PRE were propensity score matched and evaluated in the 6 months prior to and 6 months after initiation. When adjusting for potential confounders, this study found no statistically significant difference between the DUL and PRE in opioid utilization changes. Both the Gore et al and Margolis et al studies were funded by Pfizer, Inc.

Overall, these studies showed that there is no difference between DUL and PRE in opioid utilization. Additionally, they further the evidence that starting DUL does not reduce the use of opioids in patients who may already be using opioids (Table 2).

\section{MPR}

MPR is a measure of medication adherence using prescription claims to examine when and how frequently patients refill their medications. ${ }^{37}$ MPR is the ratio of the days' supply for all prescription claims during the study period divided by the number of days elapsed during the period. A patient with perfect adherence would have an MPR of 1.0, or $100 \%$. Two studies utilized MPR as a primary measure of effectiveness. ${ }^{31,32}$ As mentioned above in the DUL vs standard of care medications section, Zhao et al sub-classified patients by MPR above or below 0.8 , but did not include MPR as an outcome measure. ${ }^{30}$

\section{DUL vs PRE, TCAs, or GABA}

In 2011, Zhao et al performed a historical cohort study and found DUL patients to have significantly better adherence than PRE patients (mean MPR 0.343 vs $0.129, P<0.05$ ). ${ }^{32}$ However, in this study both groups demonstrated poor adherence, with only $15.5 \%$ and $<1 \%$ of the DUL and PRE groups, respectively, achieving $\geq 0.8$ MPR. In 2012, Oladapo et al compared MPR of both oral antidiabetic medications and DPNP medications among Texas Medicaid recipients. ${ }^{31}$ In this study DUL patients had better adherence than TCAs, GABA, or PRE in a pair-wise comparison ( 0.86 vs 0.76 TCA, 0.74 GABA, 0.69 PRE, $P<0.001$ for each). The adherence rates in this study showed a stark increase in all groups compared with the adherence rates in the Zhao et al ${ }^{32}$ study. This may be partly explained by the difference in patient populations. Zhao et al was comparing a commercially insured population, while Oladapo et $\mathrm{al}^{31}$ was examining a Medicaid population. It is unlikely that this distinction fully explains such a large discrepancy in adherence rates.

Overall, it appears DUL may have better adherence than other agents, but more research is needed to confirm these findings given the discrepancy found in these studies.

\section{Health care costs and utilization}

Of the nine real-world studies reviewed, eight included either health resource utilization or health care costs as a study endpoint. However, only three studies examined resource utilization and costs as a primary endpoint. ${ }^{33,35,36}$ All of these compared DUL to PRE. Among studies that have examined costs as a secondary endpoint, 12-month post-index mean costs (in USD) ranged from about $\$ 19,000$ to $\$ 44,000$, with most studies reporting mean total costs around $\$ 30,000$ (Table 3).

\section{DUL vs PRE}

Burke et al described changes in all-cause health care costs, DPNP-related health care costs, and health care utilization between DUL and PRE. ${ }^{33}$ All analyses were made within and between treatment groups prior to, and after initiating treatment. In both DUL and PRE, unadjusted pharmacy costs were the only all-cause costs to be significantly different from before starting treatment compared to after (DUL: $\$ 2,499$ vs $\$ 3,480, P<0.001$; PRE: $\$ 2,349$ vs $\$ 3,058, P<0.001)$. No category of all-cause costs were statistically different between DUL and PRE. Among DPNP-specific costs, both DUL and PRE unadjusted pharmacy costs were again different prior to and after treatment (DUL: $\$ 140$ vs $\$ 781, P<0.001$; PRE: $\$ 149$ vs $\$ 627, P<0.001)$. Additionally, total DPNP-related costs increased in the PRE group ( $\$ 466$ vs $\$ 1,170, P<0.001$ ). When DUL and PRE were directly compared, only the unadjusted change in DPNP-specific pharmacy costs was significantly different (DUL $\$ 641$ vs PRE $\$ 478, P=0.002$ ). Similar results were found by Margolis et al who also compared DUL to PRE in a propensity score matched cohort. ${ }^{36}$ 


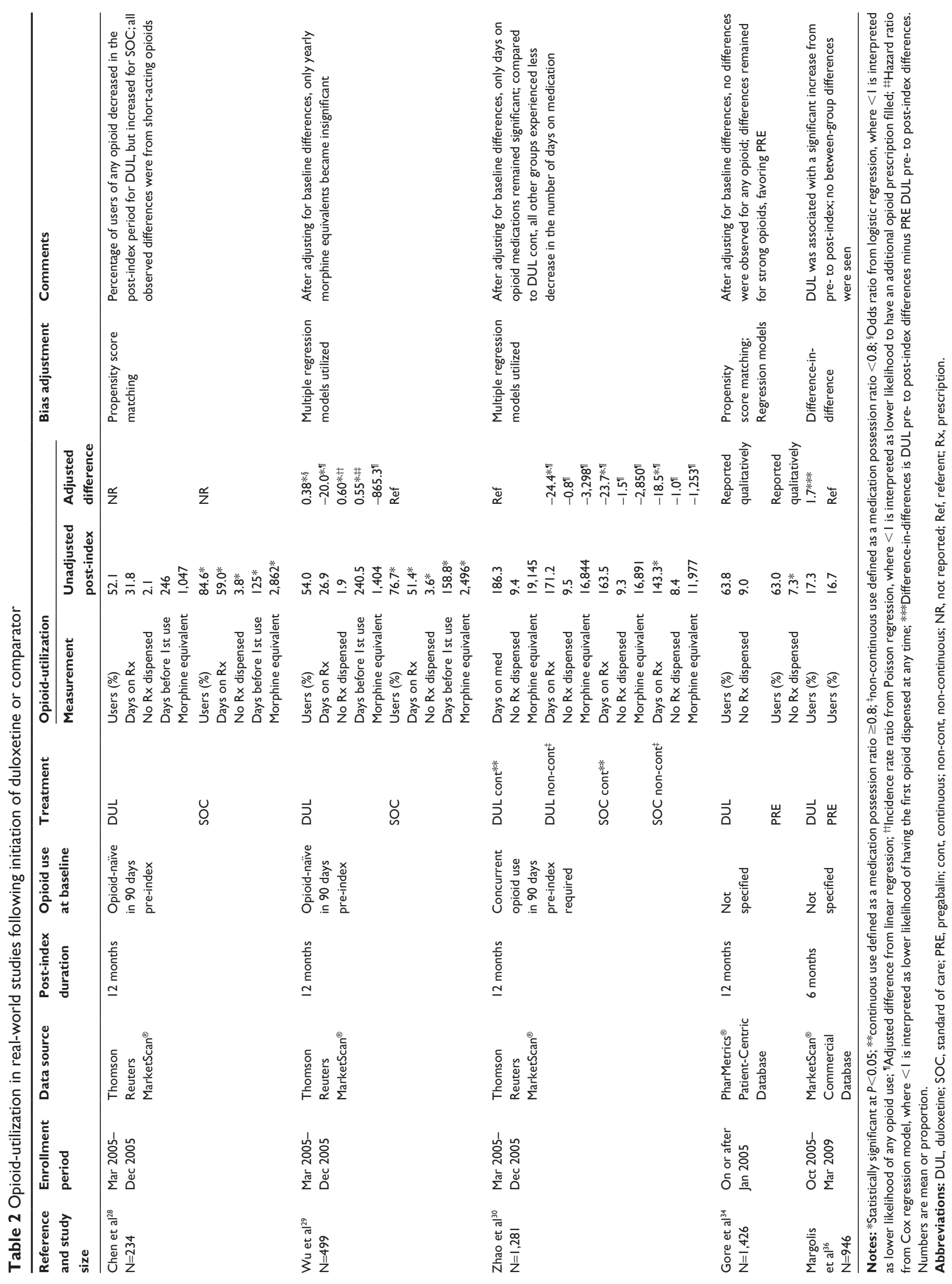




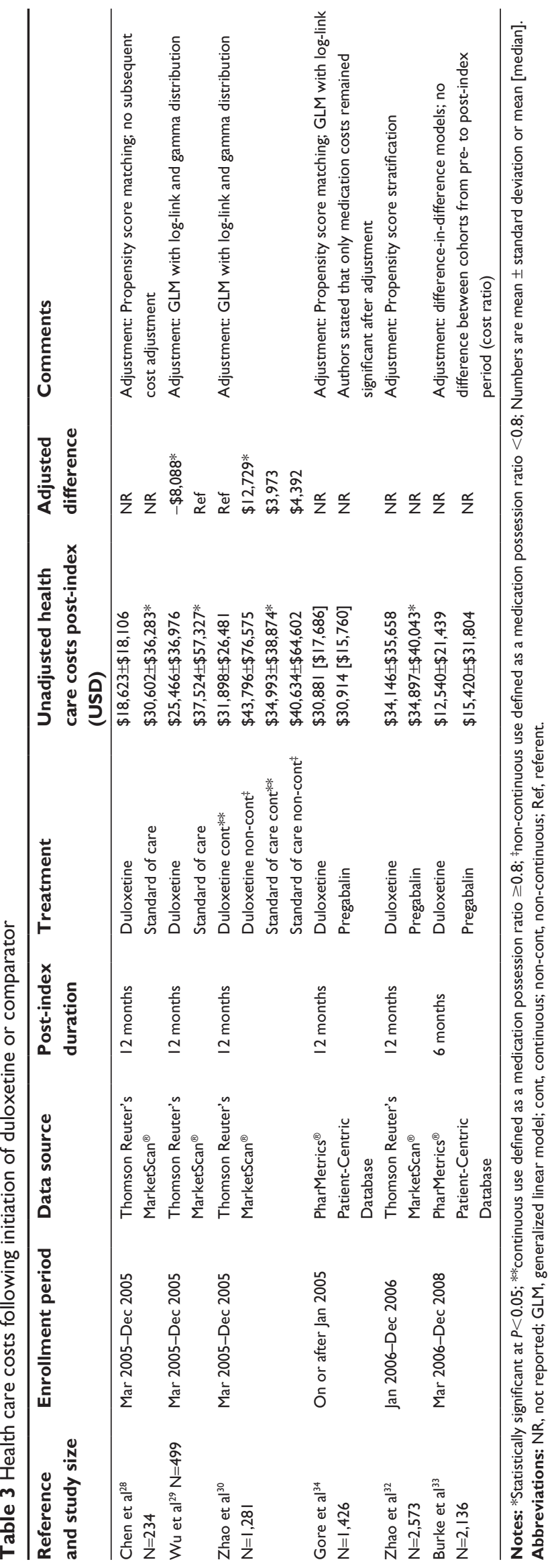

They found no significant pre to post-index period changes among all-cause or DPNP-specific health care costs.

Johnston et al compared additional costs associated with drug-drug and drug-condition interactions between DUL and PRE..$^{35}$ They found DUL to have more frequent potential drug-drug and drug-condition interactions compared with PRE (DUL $71.3 \%$ vs PRE $1.8 \%, P<0.001$ ). These potential interactions were associated with increased adjusted mean health care costs in DUL users, with potential interactions vs those without potential DUL interactions (increase: $\$ 3,346 \pm \$ 4,797, P=0.002$ ) and vs those with potential PRE interactions (increase: $\$ 6,955 \pm \$ 39,672, P=0.02$ ). Potential PRE drug-drug and drug-condition interactions were not associated with additional health care costs.

Overall, these studies showed there is a significant increase in pharmacy costs when starting treatment with DUL or PRE. It appears there is no difference in total or DPNP-related costs between DUL and PRE, but DUL may have an increase in cost due to potential drug-drug or drugcondition interactions.

\section{Pharmacoeconomic studies}

When a new drug therapy becomes available that is more effective than current alternatives, but is also more costly, decision makers must ask if the extra benefit is worth the additional costs. Pharmacoeconomics quantifies the benefits and costs of drug therapy in order to compare the value of one drug to another to help inform such decisions. Costeffectiveness analysis (CEA) is a frequently used type of pharmacoeconomic study in which value is defined as the cost per outcome. The measures of the benefits of drug therapy or outcomes in CEAs should be expressed in natural units, such as life-years, cardiovascular events, pain scores, or episodefree days. In a sub-type of CEA, called cost-utility analysis, the outcomes examined take into consideration utility values, or a patient's preference for a given health state. In the vast majority of cost-utility analysis, the benefit of drug therapy is measured in quality-adjusted life-years (QALYs), which takes into account both the quantity and quality of life.

To determine if a drug therapy is cost-effective, it needs to be compared to another treatment. The primary endpoint of interest from CEA is usually the incremental cost-effectiveness ratio (ICER). An ICER is calculated as the change in cost between two treatments divided by the change in effectiveness between them [ie, ICER $=($ cost drug A - cost drug B)/(effectiveness drug A - effectiveness drug B)]. An ICER is interpreted as the cost per outcome gained (eg, relative to drug B, drug A costs \$25,000/life-year 
gained). A therapy is deemed cost-effective if the ICER is below the willingness-to-pay (WTP) for the outcome being examined. In the United States, a commonly used WTP threshold is $\$ 50,000 / \mathrm{QALY}$. Therefore, if a therapy has an ICER $<\$ 50,000 /$ QALY gained, it may be considered cost-effective. However, this $\$ 50,000 / \mathrm{QALY}$ threshold is somewhat arbitrary and some consider WTP thresholds of $\$ 100,000-\$ 150,000 /$ QALY or even higher to be more appropriate. ${ }^{38,39}$ If a therapy costs less and is more effective, it is said that it dominates the other therapy.

\section{CEA}

The literature search identified five CEAs in patients with DPNP and included DUL as a comparator (Table 4). ${ }^{40-44}$ These studies were published between 2006 and 2012. The studies considered the cost-effectiveness of DUL from third-party payer, ${ }^{40,42,43}$ national payer, ${ }^{41,44}$ societal, ${ }^{40}$ and employer ${ }^{40}$ perspectives from the United States, ${ }^{40,42,43}$ United Kingdom, ${ }^{41}$ and Mexico. ${ }^{44}$ The time horizons used in the studies varied from 3 months, ${ }^{42,44}$ to 6 months, ${ }^{41,43}$ to 50 -weeks. ${ }^{40}$ The majority of the studies identified used decision tree models with probabilities derived from the published literature ${ }^{41-44}$ but one study was performed alongside a 52-week, open-label extension of 233 patients who completed a 12 -week RCT. ${ }^{40}$ Of the four studies which used decision trees, two reported results per 1,000 patients ${ }^{41,44}$ and the other two reported averages per patient. ${ }^{42,43}$ Additionally, the decision tree studies all used QALYs as the effectiveness outcome ${ }^{41-44}$ and the other study used the Medical Outcomes Study Short Form 36 Bodily Pain scale (SF-36 BP) score..$^{40}$ A complete review of all pharmacoeconomic studies can be found in Table S4.

\section{DUL vs PRE, GABA, or TCA}

Of the five CEAs identified, three compared DUL to PRE, GABA, or a TCA. ${ }^{42-44}$ O'Connor et al compared using DUL, PRE, GABA, and desipramine (DES) as first-line therapy in patients with DPNP. ${ }^{42}$ This study found that using either DUL or DES first-line dominated both PRE and GABA. When compared to DES, total direct medical costs were $\$ 107$ more for DUL. DUL also added an additional 0.12 QALYs, resulting in an ICER of \$47,700/QALY gained. Bellows et al used a similar tree structure to compare using DUL to PRE as first-line therapy. ${ }^{43}$ The authors found that DUL dominated PRE as it cost less (-\$187) and was more effective (additional 0.011 QALYs).

Carlos et al compared DUL to PRE, generic GABA, and branded GABA. ${ }^{44}$ This study found that DUL had lower costs than PRE ( $\$ 85,920$ per 1,000 patients) and branded GABA ( $\$ 80,080$ per 1,000 patients). However, the study did not report either the incremental costs of DUL compared to generic GABA or the incremental effectiveness of DUL compared to any other therapy. It was reported that DUL dominated both PRE and GABA, and the ICER for DUL vs generic GABA was \$8,194/QALY gained.

Overall, DUL either dominated or was a cost-effective option when compared to PRE, GABA, or DES. Generalizability was strengthened by the inclusion of all relevant comparators, ${ }^{42}$ real-world estimates of adherence, ${ }^{43}$ and concomitant therapy. ${ }^{43}$ However, none of the models included all desirable characteristics and these studies may lack generalizability due to exclusion of serious adverse events for DUL, ${ }^{42}$ inability of patients to receive treatment after discontinuation of initial treatment, ${ }^{42-44}$ and lack of inclusion of all relevant comparators. ${ }^{43,44}$

\section{DUL vs routine care medications}

Wu et al compared DUL to routine care, ${ }^{40}$ where routine care was determined by the patient and investigator and included neuropathic pain therapies excluding DUL. ${ }^{45}$ As the routine care group was somewhat ill defined and the cost of DUL was unknown, the base-case analysis excluded drug costs. The study used bootstrapping techniques to estimate $P$-values and suggested a WTP of \$100/unit of SF-36 BP. This study found that, compared to routine care, DUL resulted in a significant increase in SF-36 BP score of 6.43 points $(P=0.047)$, and nonsignificant lower costs from each perspective (payer $-\$ 1,600$, $P=0.30$; employer $-\$ 2,196, P=0.10$; societal $-\$ 2754$, $P=0.10)$. Thus DUL was dominant, but only significantly so for the societal $(P=0.04)$, and employer $(P=0.04)$ perspectives. From the payer perspective, DUL was neither dominant $(P=0.11)$, nor cost-effective $(P=0.06)$.

Overall, it is difficult to draw conclusions from this study because it excluded drug costs in the base-case scenario, and appropriate WTP values for the cost per one-unit increase in SF-36 BP score are unknown.

\section{Order of DUL treatment}

Beard et al compared when DUL was as used first-, second-, third-, or fourth-line therapy to a regimen without DUL. ${ }^{41}$ Each of the regimens used a TCA, GABA, then opioids in that order, with DUL added where indicated. The study found that using DUL as second-line therapy dominated the no DUL regimen as well as using DUL third-line or fourth-line. Using DUL first-line cost GB£42,281 more in direct medical care (per 1,000 patients), but also resulted in 


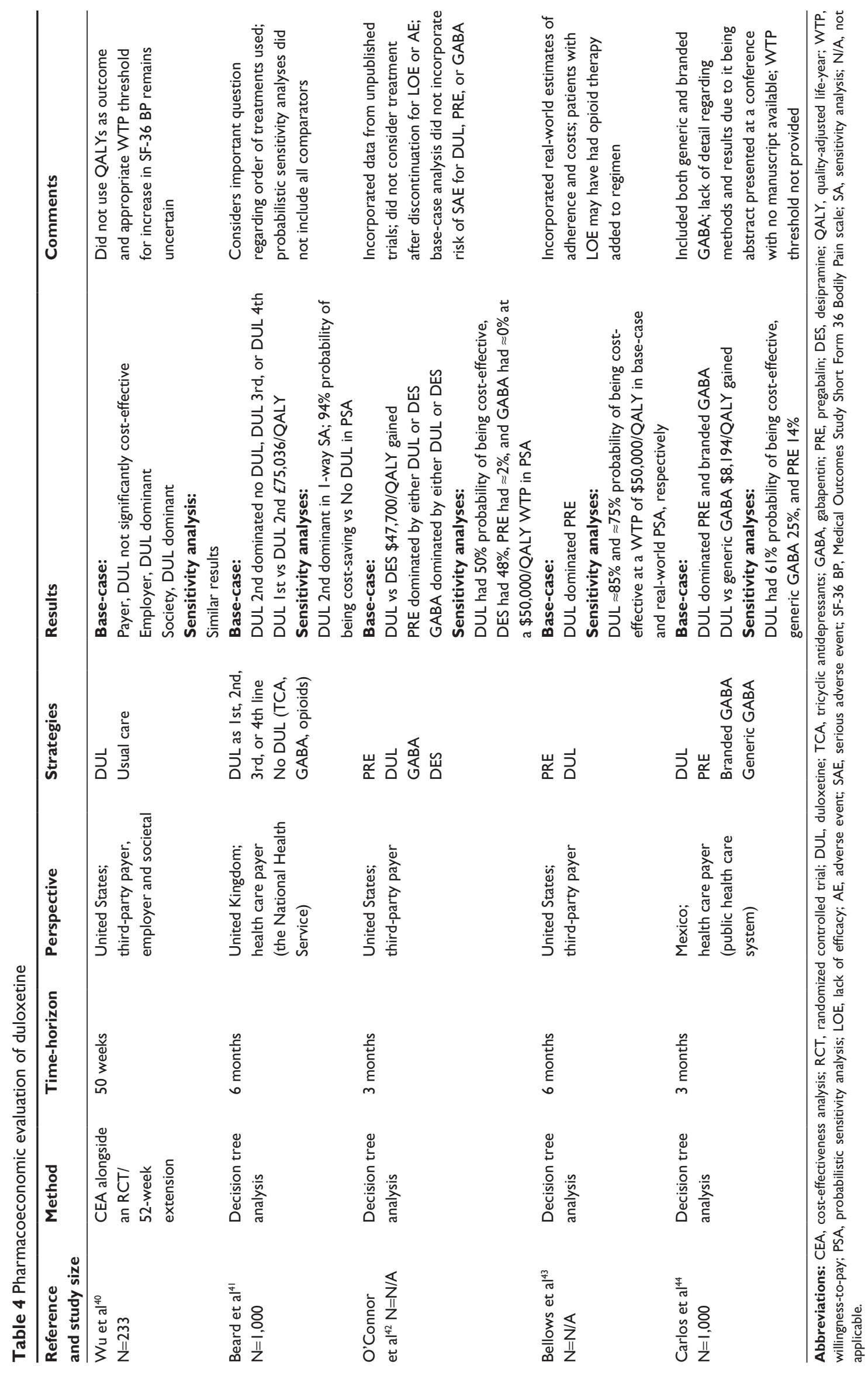


0.56 additional QALYs (per 1,000 patients) than using it second-line, resulting in an ICER of $£ 75,036 /$ QALY gained. In the United Kingdom, the National Institute for Health and Care Excellence generally considers therapies with an ICER of $£ 20,000-£ 30,000 /$ QALY gained cost-effective.

Overall, using DUL as a second-line therapy may be a preferred strategy, but more research is needed to examine the cost-effectiveness of this strategy compared other potential treatment orders.

\section{Discussion}

Diabetes mellitus is a worldwide epidemic associated with significant comorbidities and complications. Among these comorbidities, DPNP remains a highly common and challenging condition to treat. Several drugs have been established as clinically efficacious in alleviating painful symptoms and, based on clinical trial evidence, DUL, TCAs, PRE, and GABA are all considered an appropriate first-line therapy for the treatment of DPNP. ${ }^{11,46}$ This review has focused on the clinical utility of DUL compared to other drugs in DPNP using many available sources of evidence.

In clinical trials, DUL was generally well tolerated and was efficacious for reducing pain scores when the daily dose was $\geq 60 \mathrm{mg}$. However, head-to-head comparisons with other relevant treatments for DPNP showed mixed results. In two trials of DUL vs AMI, both medications were found to significantly reduce pain scores from baseline, but no significant differences were found between the two. ${ }^{25,26}$ Similarly, when compared with PRE, the only other medication currently FDA approved for the treatment of DPNP, studies were conflicting on an increased benefit associated with DUL. ${ }^{21,22,25}$ Hence, it cannot be concluded in this review if one drug is better than the other at reducing DPNP-related pain in a randomized controlled environment.

Reductions in opioid utilization, medication adherence, and health care costs were the most commonly used measures of effectiveness in real-world studies. When compared with any DPNP medication, DUL use was associated with less opioid utilization after starting treatment in patients who were not currently receiving opioids. ${ }^{28,29}$ However, in patients who were receiving opioids at the time of initiation of DUL or other DPNP medications, there was no significant difference in overall opioid utilization. ${ }^{30}$ Additionally, the comparator group in these studies included medications that are considered both first-line and second-line, which may not be the most appropriate comparison. When DUL was compared directly to PRE, no significant pre-to-post differences were observed in DPNP-related analgesic medication use. ${ }^{36}$ In separate populations, DUL has shown significantly better adherence than PRE, GABA, or TCAs. ${ }^{31,32}$ Total all-cause and DPNP-associated health care costs estimated using insurance claims databases were generally similar between patients initiated on DUL and PRE. ${ }^{32-34,36}$

Despite the variety of methodologies used, DUL was consistently found to be a cost-effective option in treating DPNP ${ }^{40-44}$ Decision makers may consider using this evidence to guide treatment and coverage decisions accordingly. However, DPNP is a chronic disease and patients will likely be treated for extended periods of time. The long-term costeffectiveness of DUL remains unknown and future CEAs should consider longer timeframes. Additionally, optimal treatment pathways have not been established and future analyses should focus on finding the most cost-effective treatment pathways for patients. While Beard et al began to assess this important question, their analysis was limited in that treatments other than DUL were used in the same order. ${ }^{41}$ Finally, combinations of treatments may be used to treat DPNP in clinical practice settings and studies should consider the impact of specific combinations of treatments.

There are many challenges to using clinical utility data from clinical trials, real-world studies, and pharmacoeconomic studies in making health care decisions. One of these challenges is that safety and efficacy data from clinical trials, as well as personal experience, have been important factors in physicians' assessments of clinical utility. ${ }^{47}$ However, personal experience can be anecdotal and clinical trials are designed to determine the efficacy of an intervention, or the benefit of that intervention under ideal conditions. Well-designed clinical trials are costly, time-consuming, and may over-state the benefit that patients may expect in a non-controlled environment. Real-world research set outs to establish effectiveness, or how well an intervention performs outside the realm of homogenous patient populations and rigorously controlled conditions, however, it is subject to inherent biases. These biases must be addressed in the study design and statistical analyses of real-world studies, or the results may not represent true effectiveness. Where clinical trials tend to be more easily understood, real-world and pharmacoeconomic studies are layered with complex statistical methods, which make interpretation, let alone critical evaluation, challenging for decision makers without training in the evaluation of real-world and pharmacoeconomic literature. Without this background knowledge, it is often difficult to know whether or not these procedures have been applied appropriately. 
There is a significant delay between the availability of a new product and the availability of real-world data from clinical practice. For example, DUL was first approved by the FDA in 2004, however, the first real-world comparativeeffectiveness study identified in this review was published in 2010. Further, once data is available it is challenging to make clinical or cost predictions beyond the limited timeframe, an issue which commonly arises in economic modeling studies.

Complicating this issue is the role the pharmaceutical industry plays in funding the majority of real-world and pharmacoeconomic studies, which leads to some distrust in the validity of the results. Of the 13 real-world and pharmacoeconomic studies reported in this review, ten received funding from the pharmaceutical industry, one was funded by an NIH grant, and two were unfunded. Ultimately, the evidence derived from research is only as effective as the decision maker's ability to interpret and apply that evidence effectively. Unfortunately, there is a significant lack of education around non-randomized research methods in medical schools. This is a burden faced by clinicians, managed care organizations, and well-meaning researchers. There is a need for improved education of real-world and pharmacoeconomic research methodologies.

There are additional challenges to non-clinical trial research that are specific to pain related conditions. As previously mentioned, the measures used in clinical trials to evaluate the efficacy of treatment are rarely found in real-world databases. As an alternative, researchers have used surrogate markers of effectiveness such as opioid utilization, medication adherence, and health care costs or resource utilization. These endpoints are generally considered comparable markers of clinical effectiveness, however, they should be interpreted cautiously; surrogate endpoints can misrepresent the true effect of an intervention. In a retrospective review, they are also more prone to misclassification. Also, severity of DPNP, which has been found to be a significant predictor of health care costs and utilization, is unable to be assessed in a claims database further complicating this research.

Based on the review of the current clinical utility data available for DUL, there is sufficient evidence to support its use as a first-line treatment option from the perspective of patients, clinicians, and managed care payers. However, further research is needed on the optimal treatment algorithm for DPNP, including medication sequencing and combination therapy. Also, evidence to support the long-term clinicaleffectiveness and cost-effectiveness of DUL is lacking.

\section{Disclosure}

The authors have no conflicts of interest to declare in this work.

\section{References}

1. Centers for Disease Control and Prevention [webpage on the Internet]. National Diabetes Statistics Report: Estimates of Diabetes and Its Burden in the United States A, GA: US Department of Health and Human Services, 2014. Available from: http://www.cdc.gov/diabetes/ data/statistics/2014statisticsreport.html. Accessed April 2, 2015.

2. Barrett AM, Lucero MA, Le T, Robinson RL, Dworkin RH, Chappell AS. Epidemiology, public health burden, and treatment of diabetic peripheral neuropathic pain: a review. Pain Med. 2007;8 Suppl 2: S50-S62.

3. Davies M, Brophy S, Williams R, Taylor A. The prevalence, severity, and impact of painful diabetic peripheral neuropathy in type 2 diabetes. Diabetes Care. 2006;29(7):1518-1522.

4. National Institute of Diabetes and Digestive and Kidney Diseases [webpage on the Internet]. Diabetic Neuropathies: The Nerve Damage of Diabetes, 2013. Available from: http://diabetes.niddk.nih.gov/dm/ pubs/neuropathies/. Accessed April 2, 2015.

5. Boulton AJ, Vinik AI, Arezzo JC, et al. Diabetic neuropathies: a statement by the American Diabetes Association. Diabetes Care. 2005;28(4):956-962.

6. Galer BS, Gianas A, Jensen MP. Painful diabetic polyneuropathy: epidemiology, pain description, and quality of life. Diabetes Res Clin Pract. 2000;47(2):123-128.

7. [No authors listed]. Standards of medical care in diabetes - 2015: summary of revisions. Diabetes Care. 2015;38 Suppl:S4.

8. Callaghan BC, Little AA, Feldman EL, Hughes RA. Enhanced glucose control for preventing and treating diabetic neuropathy. Cochrane Database Syst Rev. 2012;6:CD007543.

9. Ziegler D, Fonseca V. From guideline to patient: a review of recent recommendations for pharmacotherapy of painful diabetic neuropathy. J Diabetes Complications. 2015;29(1):146-156.

10. American Diabetes Association. (9) Microvascular complications and foot care. Diabetes Care. 2015;38 Suppl:S58-S66.

11. Bril V, England J, Franklin GM, et al. Evidence-based guideline: Treatment of painful diabetic neuropathy: report of the American Academy of Neurology, the American Association of Neuromuscular and Electrodiagnostic Medicine, and the American Academy of Physical Medicine and Rehabilitation. Neurology. 2011;76(20): 1758-1765.

12. Handelsman Y, Mechanick JI, Blonde L, et al. American Association of Clinical Endocrinologists Medical Guidelines for Clinical Practice for developing a diabetes mellitus comprehensive care plan. Endocr Pract. 2011;17 Suppl 2:1-53.

13. Lesko LJ, Zineh I, Huang SM. What is clinical utility and why should we care? Clin Pharmacol Ther. 2010;88(6):729-733.

14. Gao Y, Ning G, Jia WP, et al. Duloxetine versus placebo in the treatment of patients with diabetic neuropathic pain in China. Chin Med J (Engl). 2010;123(22):3184-3192.

15. Goldstein DJ, Lu Y, Detke MJ, Lee TC, Iyengar S. Duloxetine vs placebo in patients with painful diabetic neuropathy. Pain. 2005; 116(1-2):109-118.

16. Raskin J, Pritchett YL, Wang F, et al. A double-blind, randomized multicenter trial comparing duloxetine with placebo in the management of diabetic peripheral neuropathic pain. Pain Med. 2005;6(5): 346-356.

17. Raskin J, Smith TR, Wong K, et al. Duloxetine versus routine care in the long-term management of diabetic peripheral neuropathic pain. J Palliat Med. 2006;9(1):29-40.

18. Raskin J, Wang F, Pritchett YL, Goldstein DJ. Duloxetine for patients with diabetic peripheral neuropathic pain: a 6-month open-label safety study. Pain Med. 2006;7(5):373-385. 
19. Rowbotham MC, Arslanian A, Nothaft W, et al. Efficacy and safety of the alpha4beta2 neuronal nicotinic receptor agonist ABT-894 in patients with diabetic peripheral neuropathic pain. Pain. 2012;153(4): $862-868$

20. Skljarevski V, Desaiah D, Zhang Q, et al. Evaluating the maintenance of effect of duloxetine in patients with diabetic peripheral neuropathic pain. Diabetes Metab Res Rev. 2009;25(7):623-631.

21. Tanenberg RJ, Irving GA, Risser RC, et al. Duloxetine, pregabalin, and duloxetine plus gabapentin for diabetic peripheral neuropathic pain management in patients with inadequate pain response to gabapentin: an open-label, randomized, noninferiority comparison. Mayo Clin Proc. 2011;86(7):615-626.

22. Tesfaye S, Wilhelm S, Lledo A, et al. Duloxetine and pregabalin: high-dose monotherapy or their combination? The "COMBO-DN study" - a multinational, randomized, double-blind, parallel-group study in patients with diabetic peripheral neuropathic pain. Pain. 2013;154(12):2616-2625.

23. Wernicke JF, Pritchett YL, D'Souza DN, et al. A randomized controlled trial of duloxetine in diabetic peripheral neuropathic pain. Neurology. 2006;67(8):1411-1420.

24. Wernicke JF, Wang F, Pritchett YL, et al. An open-label 52-week clinical extension comparing duloxetine with routine care in patients with diabetic peripheral neuropathic pain. Pain Med. 2007;8(6):503-513.

25. Boyle J, Eriksson ME, Gribble L, et al. Randomized, placebo-controlled comparison of amitriptyline, duloxetine, and pregabalin in patients with chronic diabetic peripheral neuropathic pain: impact on pain, polysomnographic sleep, daytime functioning, and quality of life. Diabetes Care. 2012;35(12):2451-2458.

26. Kaur H, Hota D, Bhansali A, Dutta P, Bansal D, Chakrabarti A. A comparative evaluation of amitriptyline and duloxetine in painful diabetic neuropathy: a randomized, double-blind, cross-over clinical trial. Diabetes Care. 2011;34(4):818-822.

27. Annemans L, Aristides M, Kubin M. Real-Life Data: A Growing Need. ISPOR Connections. 2007;13(5):8-12.

28. Chen S, Wu N, Fraser K, Boulanger L, Zhao Y. Opioid use and healthcare costs among patients with DPNP initiating duloxetine versus other treatments. Curr Med Res Opin. 2010;26(10):2507-2516.

29. Wu N, Chen SY, Hallett LA, et al. Opioid utilization and health-care costs among patients with diabetic peripheral neuropathic pain treated with duloxetine vs other therapies. Pain Pract. 2011;11(1):48-56.

30. Zhao Y, Wu N, Chen S, Boulanger L, Police RL, Fraser K. Changes in opioid use and healthcare costs among U.S. patients with diabetic peripheral neuropathic pain treated with duloxetine compared with other therapies. Curr Med Res Opin. 2010;26(9):2147-2156.

31. Oladapo AO, Barner JC, Rascati KL, Strassels SA. A retrospective database analysis of neuropathic pain and oral antidiabetic medication use and adherence among Texas adults with type 2 diabetes enrolled in Medicaid. Clin Ther. 2012;34(3):605-613.

32. Zhao Y, Sun P, Watson P. Medication adherence and healthcare costs among patients with diabetic peripheral neuropathic pain initiating duloxetine versus pregabalin. Curr Med Res Opin. 2011;27(4):785-792.
33. Burke JP, Sanchez RJ, Joshi AV, Cappelleri JC, Kulakodlu M, Halpern R. Health care costs in patients with painful diabetic peripheral neuropathy prescribed pregabalin or duloxetine. Pain Pract. 2012;12(3):209-218.

34. Gore M, Zlateva G, Tai KS, Chandran AB, Leslie D. Retrospective evaluation of clinical characteristics, pharmacotherapy and healthcare resource use among patients prescribed pregabalin or duloxetine for diabetic peripheral neuropathy in usual care. Pain Pract. 2011;11(2): 167-179.

35. Johnston SS, Udall M, Cappelleri JC, et al. Cost comparison of drugdrug and drug-condition interactions in patients with painful diabetic peripheral neuropathy treated with pregabalin versus duloxetine. Am J Health Syst Pharm. 2013;70(24):2207-2217.

36. Margolis J, Cao Z, Fowler R, et al. Evaluation of healthcare resource utilization and costs in employees with pain associated with diabetic peripheral neuropathy treated with pregabalin or duloxetine. $\mathrm{J} \mathrm{Med}$ Econ. 2010;13(4):738-747.

37. Steiner JF, Prochazka AV. The assessment of refill compliance using pharmacy records: methods, validity, and applications. J Clin Epidemiol. 1997;50(1):105-116.

38. Neumann PJ, Cohen JT, Weinstein MC. Updating cost-effectiveness the curious resilience of the \$50,000-per-QALY threshold. $N$ Engl J Med. 2014;371(9):796-797.

39. Braithwaite RS, Meltzer DO, King JT Jr, Leslie D, Roberts MS. What does the value of modern medicine say about the $\$ 50,000$ per qualityadjusted life-year decision rule? Med Care. 2008;46(4):349-356.

40. Wu EQ, Birnbaum HG, Mareva MN, et al. Cost-effectiveness of duloxetine versus routine treatment for U.S. patients with diabetic peripheral neuropathic pain. J Pain. 2006;7(6):399-407.

41. Beard SM, McCrink L, Le TK, Garcia-Cebrian A, Monz B, Malik RA. Cost effectiveness of duloxetine in the treatment of diabetic peripheral neuropathic pain in the UK. Curr Med Res Opin. 2008; 24(2):385-399.

42. O'Connor AB, Noyes K, Holloway RG. A cost-utility comparison of four first-line medications in painful diabetic neuropathy. Pharmacoeconomics. 2008;26(12):1045-1064.

43. Bellows BK, Dahal A, Jiao T, Biskupiak J. A cost-utility analysis of pregabalin versus duloxetine for the treatment of painful diabetic neuropathy. J Pain Palliat Care Pharmacother. 2012;26(2):153-164.

44. Carlos F, Ramírez-Gámez J, Dueñas H, Galindo-Suárez RM, Ramos E. Economic evaluation of duloxetine as a first-line treatment for painful diabetic peripheral neuropathy in Mexico. $J$ Med Econ. 2012;15(2):233-244.

45. Dworkin RH, Backonja M, Rowbotham MC, et al. Advances in neuropathic pain: diagnosis, mechanisms, and treatment recommendations. Arch Neurol. 2003;60(11):1524-1534.

46. Tan T, Barry P, Reken S, Baker M; Guideline Development Group. Pharmacological management of neuropathic pain in non-specialist settings: summary of NICE guidance. BMJ. 2010;340:c1079.

47. Schumock GT, Walton SM, Park HY, et al. Factors that influence prescribing decisions. Ann Pharmacother. 2004;38(4):557-562.
Therapeutics and Clinical Risk Management

\section{Publish your work in this journal}

Therapeutics and Clinical Risk Management is an international, peerreviewed journal of clinical therapeutics and risk management, focusing on concise rapid reporting of clinical studies in all therapeutic areas, outcomes, safety, and programs for the effective, safe, and sustained use of medicines. This journal is indexed on PubMed Central, CAS,

\section{Dovepress}

EMBase, Scopus and the Elsevier Bibliographic databases. The manuscript management system is completely online and includes a very quick and fair peer-review system, which is all easy to use. Visit http://www.dovepress.com/testimonials.php to read real quotes from published authors. 